\title{
Deprivation and prognosis in patients with pulmonary arterial hypertension: missing the effect of deprivation on a rare disease?
}

To the Editor:

In this journal, Pellino et al. [1] presented a survival analysis to assess how deprivation affects prognosis in patients with pulmonary arterial hypertension (PAH). Their conclusions were that social deprivation is not a significant referral barrier or prognostic factor for idiopathic (I)PAH or heritable (H)PAH in Scotland. This may appear surprising, given the wider context of literature describing outcomes stratified by social deprivation. The authors were thorough on using both the address at time of diagnosis and at time of censoring to assign deprivation scores and compare the two, finding no significant differences between the two approaches. They also compared deprivation assigned to PAH cases to expected deprivation based on Scottish citizenry as a whole, and found that PAH patients are more socially deprived than expected. Finally, they used the same survival univariate analysis adjusting for age and sex to assess how several clinical variables are associated with prognosis.

The ongoing National Cohort Study of Idiopathic and Heritable PAH collects data from all of the UK and offers an opportunity to test the relationship between deprivation and outcomes in PAH in a second population that is geographically distinct but in many other ways highly analogous to that studied by Pellino et al. [1]. The study collects data from a contemporaneous cohort in all of the specialist PAH centres in the UK. Therefore, we were able to assess mortality in relation to deprivation in $270 \mathrm{PAH}$ patients living in England and Wales, residing in the same addresses since diagnosis. Our patients were recruited in the UK PAH cohort from 1 January, 2014 and followed-up until 28 February, 2019. 36 (13\%) participants died and five (2\%) were transplanted, resulting in 1-, 3- and 5-year transplant-free survival estimates of $96 \%, 86 \%$ and $73 \%$ for incident patients $(n=146)$ and $100 \%, 100 \%$ and $89 \%$ for prevalent patients ( $\mathrm{n}=124)$, respectively. Pellino et al. [1] captured deprivation using the Scottish Index of Multiple Deprivation (IMD), which is a composite index comprising of sub-indices (e.g. income, education, employment, health, access to services, crime). In the UK, IMD is not comparable between England, Scotland and Wales, so for our UK cohort we accessed an adjusted IMD index for use across the UK [2], which consists of the three main domains of IMD: income, employment and educational deprivation. In addition to the area level deprivation captured via the UK-IMD, the primary survival model was adjusted for age at diagnosis and sex, to be in line with the adjustment used by Pellino et al. [1], fitting Cox proportional hazards regression models. Cox proportional hazards regression models and survival curves were fitted allowing for left truncation arising from the interval between diagnosis of prevalent cases and enrolment. Prevalent patients were only included in the risk set from the time of study entry and were excluded if they entered the study $>10$ years after diagnosis. We stratified the analysis by centre, due to the multicentre design of our study. In the extended model, we further adjusted for smoking, prevalent/ incident status and risk profiles (low/intermediate/high) at the time of diagnosis, calculated using the abbreviated 2015 European Society of Cardiology (ESC)/European Respiratory Society (ERS) risk stratification strategy [3]. According to data availability, the risk score groups were estimated using the World Health Organization functional class, right atrial pressure, cardiac index and mixed venous oxygen saturation, having significant difference $(\mathrm{p}=0.02)$ on survival (figure 1 ), as expected.

@ERSpublications

No association was found between deprivation and mortality for PAH patients in England and Wales. The association found between risk stratification at baseline and deprivation suggests that the issue of deprivation and outcomes in PAH may be more nuanced. https://bit.ly/2y $8 \mathrm{WgqB}$

Cite this article as: Sofianopoulou E, Church C, Coghlan G, et al. Deprivation and prognosis in patients with pulmonary arterial hypertension: missing the effect of deprivation on a rare disease? Eur Respir $J$ 2020; 56: 1902334 [https://doi.org/10.1183/13993003.02334-2019]. 


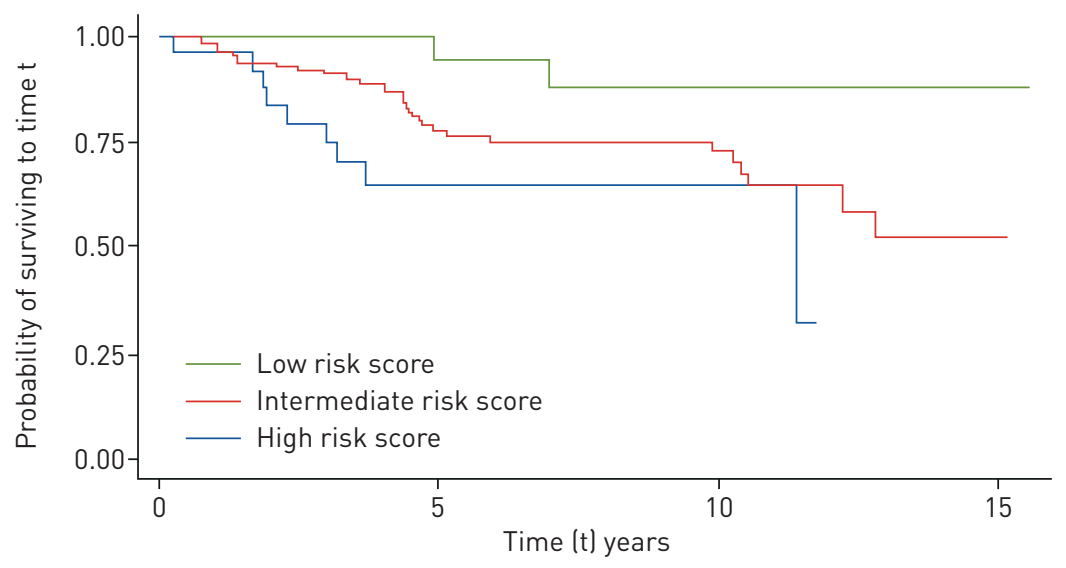

At risk $\mathrm{n}$

Low risk score 16

Intermediate risk score $\quad 104$

High risk score

16

26

17
61

9

30

3
1

2

0

FIGURE 1 Transplant-free survival in relation to risk profile (Kaplan-Meier estimates), at the time of diagnosis $(n=270)$.

The study participants are more likely to be women (68\%) with mean \pm SD age at diagnosis $51 \pm 16$ years. The pulmonary haemodynamic characteristics of the study participants were: 1) right atrial pressure $9 \pm 5 \mathrm{mmHg}$, 2) mean pulmonary arterial pressure, $53 \pm 14 \mathrm{mmHg}, 3)$ cardiac index $2 \pm 0.6 \mathrm{~L} \cdot \mathrm{min}^{-1} \cdot \mathrm{m}^{-2}$, 4) cardiac output $\left.4 \pm 1.2 \mathrm{~L} \cdot \mathrm{min}^{-1}, 5\right)$ pulmonary vascular resistance $12 \pm 5 \mathrm{WU}$ and 6) mixed venous oxygen saturation, $64 \pm 8 \%$. We found no association between mortality and classes of area deprivation, for both the primary and adjusted model. As an example, adjusted hazard ratio (HR) was 0.63 (95\% CI 0.18-2.16) ( $p=0.458$ ) for most deprived (quintile 5) areas compared to least deprived (quintile 1) areas. To examine this further, we investigated area deprivation as predictor of risk profiles, fitting multinomial regression models. We found an association between risk profiles and area deprivation, while adjusting for the same variables as above. Living within the more deprived areas (quintiles 5, 4 and 3) was associated with higher $\mathrm{PAH}$ risk score at baseline, compared to the least deprived areas (quintile 1), therefore increasing the likelihood of being diagnosed within the ESC/ERS high-risk clinical prediction category. Testing for the overall effect of deprivation to risk profiles, we found a statistically significant predictor effect $(p=0.034$, Wald-test). The relative risk ratio changing from least (quintile 1) to most (quintile 5) deprived area is 15.01 (95\% CI 1.96-114.96) ( $\mathrm{p}=0.009$ ), for being classed as high risk profile versus low risk profile. This was 14.59 (95\% CI 2.22-95.9) $(\mathrm{p}=0.005)$ and 9.75 (95\% CI 1.42-67.06) ( $\mathrm{p}=0.021)$ for quintiles 4 and 3, respectively. In other words, we found that the expected risk for being in high-risk class is higher for subjects who live in the most deprived areas. The baseline risk class was also found to be a predictor of mortality in this survival analysis (adjusted HR 6.71, 95\% CI 1.22-36.87) ( $p=0.028$ ), in line with the literature [3-5].

The notion that deprivation is associated with increased mortality and morbidity for the most deprived compared with the most affluent, is confirmed by many studies and, therefore, has been enshrined in health studies, theories and policies [6-9]. The main aspects of deprivation are income, employment and education that can be collected at area-level and individual level or, preferably, both. The choice for using a composite index or its subdomains, or individual-level deprivation, is driven by the research question and availability of deprivation indicators. Studies have faced numerous challenges in terms of using a composite area-level deprivation index. The data required are based on coverage of routinely collected data, which can vary between countries, using also a weighting score that depends on the priorities set by each country, making these indices not comparable. Usually, though, the highest weights are given to income, employment and educational deprivation and, therefore, it is possible the composite index is similar to indices that are based on any of these traditional deprivation markers. Still, we recommend not to assume that any differences would have no impact on the results, especially when using small datasets as the models can be too sensitive.

Even when such an index is used for the intended geographical area it was created for, the geographical distributions of sub-indices may have opposing directions, introducing error on the overall measure of deprivation. For instance, a high deprivation score should reflect both areas of low income and difficulty 
in accessing healthcare, with the latter often being measured as distance to primary healthcare centre. Patients who live near city centres, which tend to have higher income deprivation, may not have a long distance to access a primary care centre, while the opposite holds for wealthier populations that may live outside the city centre. Thus, the estimated composite deprivation can be compromised. Even the traditional indicators of income and education deprivation may vary substantially across areas [10]. Any differences can be understood by mapping the individual domains, which is important when considering how such data is used in analyses.

Composite IMD indices, such as the Scottish IMD used by PeLLino et al. [1], have a health subdomain calculated based on standardised mortality ratio among other variables, so PAH mortality may be captured in some degree by this sub-domain. Therefore, ideally, the health sub-domain should not be included when deprivation is examined in relation to mortality. Finally, we would argue that even when prognosis is not associated with deprivation, this cannot directly lead to the interpretation that prognosis is not affected by access to care and referrals. Deprivation is linked to several parameters that affect health (i.e. diet, air quality) other than just equity on access to health services.

A notable feature of the UK National Health Service specialised commissioning structure for PAH is the equity of access to all licensed treatments to all patients, once they have been referred to the centres. A possible interpretation of our results and those reported by PeLlino et al. [1] is therefore that deprivation acts as a barrier to referral but once in the specialised system, the equity of treatment availability diminishes the subsequent effect on mortality. We feel it cannot be ignored that, unlike previous work that showed a significant effect of deprivation on mortality [11], both UK studies of similar sample sizes have failed to demonstrate an association with mortality and in health systems with more equal access to licensed treatment.

There are some limitations to this work. It is common for rare disease projects to analyse hundreds of cases, compared to thousands or millions in common diseases, for the same or similar research questions $[12,13]$, leading to the need to adopt refined datasets and analytical methods. The introduction of measurement error when using a deprivation index is likely to affect small datasets disproportionately and needs to be used with caution when conducting sensitivity analysis. Lastly, we are unable to quantify the effect of patients potentially not surviving to first admission to a tertiary $\mathrm{PH}$ care centre, and this drop out is theoretically more significant, specifically because of the association we find with baseline risk stratification.

Overall, the study by PELLiNo et al. [1] is an important piece in the PAH aetiology puzzle, and the results of our study are in line with their lack of association between mortality and social deprivation; although we would caution that both studies are of modest size. At the same time, Pellino et al. [1] found that PAH patients are more socially deprived than expected, based on Scottish citizenry as a whole. This alludes that deprivation status may have an association with $\mathrm{PAH}$ rather than it being a random finding. Our study adds further to the literature by demonstrating that higher area deprivation is associated with a worse risk profile at baseline, which in turn is a predictor of worse survival rates, suggesting that the issue of social deprivation and outcomes in PAH may be more nuanced. Examining both primary healthcare and hospital data in PAH could provide evidence on whether in deprived areas delays on PAH diagnosis are associated with the patient taking longer to seek help and the time required for tests related to the diagnosis, compared to least deprived areas.

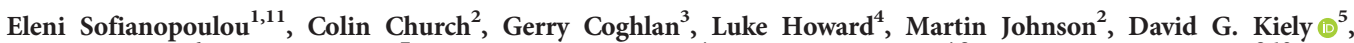
Allan Lawrie $\odot^{6}$, James Lordan ${ }^{7}$, Martin R. Wilkins $\oplus^{4}$, Stephen J. Wort ${ }^{4,8}$, Nicholas W. Morrell ${ }^{9,10}$ and Mark R. Toshner $\oplus^{9,11}$

${ }^{1}$ Dept of Public Health and Primary Care, Cardiovascular Epidemiology Unit, University of Cambridge, Cambridge, UK. ${ }^{2}$ Golden Jubilee National Hospital, Glasgow, UK. ${ }^{3}$ Royal Free Hospital, London, UK. ${ }^{4}$ Imperial College London, London, UK. ${ }^{5}$ Sheffield Pulmonary Vascular Disease Unit, Royal Hallamshire Hospital, Sheffield, UK. ${ }^{6}$ Dept of Infection, Immunity and Cardiovascular Disease, University of Sheffield, Sheffield, UK. ${ }^{7}$ University of Newcastle, Newcastle, UK. ${ }^{8}$ Royal Brompton Hospital, London, UK. ${ }^{9}$ Dept of Medicine, University of Cambridge, Cambridge, UK.

${ }^{10}$ NIHR BioResource - Rare Diseases, Cambridge, UK. ${ }^{11}$ Supervision of this work was shared by E. Sofianopoulou and M.R. Toshner.

Correspondence: Eleni Sofianopoulou, University of Cambridge, Dept of Public Health and Primary Care, Wort's Causeway, Strangeways Research Laboratory, Cambridge CB1 8RN, UK. E-mail: es630@medschl.cam.ac.uk

Received: 5 Dec 2019 | Accepted after revision: 20 March 2020

Conflict of interest: E. Sofianopoulou has nothing to disclose. C. Church has nothing to disclose. G. Coghlan reports grants and personal fees for conference attendance from Johnson \& Johnson, personal fees for lectures from GlaxoSmithKline, Bayer andMSD, outside the submitted work. L. Howard has nothing to disclose. M. Johnson reports grants and personal fees for meeting attendance, lectures and advisory board work from Actelion and MSD, outside the submitted work. D.G. Kiely has nothing to disclose. A. Lawrie reports grants from British Heart Foundation and 
Medical Research Council, grants, personal fees and travel support from Actelion Pharmaceuticals Ltd, grants and personal fees from GlaxoSmithKline, outside the submitted work. J. Lordan has nothing to disclose. M.R. Wilkins has nothing to disclose. S.J. Wort has nothing to disclose. N.W. Morrell has nothing to disclose. M.R. Toshner reports personal fees from GSK, grants and personal fees from J\&J/Actelion, grants from Merck and Bayer, outside the submitted work.

Support statement: This work was supported by the Medical Research Council (grant: RG67444), the British Heart Foundation (grant: RG68204) and the NIHR Cambridge Biomedical Research Centre. Funding information for this article has been deposited with the Crossref Funder Registry.

\section{References}

1 Pellino K, Kerridge S, Church C, et al. Social deprivation and prognosis in Scottish patients with pulmonary arterial hypertension. Eur Respir J 2018; 51: 1700444.

2 Abel GA, Barclay ME, Payne RA. Adjusted indices of multiple deprivation to enable comparisons within and between constituent countries of the UK including an illustration using mortality rates. BMJ Open 2016; 6: e012750.

3 Hoeper MM, Kramer T, Pan Z, et al. Mortality in pulmonary arterial hypertension: prediction by the 2015 European pulmonary hypertension guidelines risk stratification model. Eur Respir J 2017; 50: 1700740.

4 Sofianopoulou E, Kaptoge S, Gräf S, et al. Traffic exposures, air pollution and outcomes in pulmonary arterial hypertension: a UK cohort study analysis. Eur Respir J 2019; 53: 1801429.

5 Kylhammar D, Kjellström B, Hjalmarsson C, et al. A comprehensive risk stratification at early follow-up determines prognosis in pulmonary arterial hypertension. Eur Heart J 2018; 39: 4175-4181.

6 Stafford M, Marmot M. Neighbourhood deprivation and health: does it affect us all equally? Int J Epidemiol 2003; 32: $357-366$.

7 Carstairs V, Morris R. Deprivation and Health in Scotland. Edinburgh, Mercat Press, 1991.

8 Barnett K, Mercer SW, Norbury M, et al. Epidemiology of multimorbidity and implications for health care, research, and medical education: a cross-sectional study. Lancet 2012; 380: 37-43.

9 Office of National Statistics. Statistical Bulletin: Health State Life Expectancies by National Deprivation Deciles, England: 2016 to 2018. www.ons.gov.uk/peoplepopulationandcommunity/healthandsocialcare/healthinequalities/ bulletins/healthstatelifeexpectanciesbyindexofmultipledeprivationimd/2016to2018 Date last updated: 27 March 2020.

10 Sofianopoulou E, Rushton SP, Diggle PJ, et al. Association between respiratory prescribing, air pollution and deprivation, in primary health care. J Public Health 2013; 35: 502-509.

$11 \mathrm{Wu} \mathrm{WH}$, Yang L, Peng FH, et al. Lower socioeconomic status is associated with worse outcomes in pulmonary arterial hypertension. Am J Respir Crit Care Med 2013; 187: 303-310.

12 Woodward M, Brindle P, Tunstall-Pedoe $\mathrm{H}$. Adding social deprivation and family history to cardiovascular risk assessment: the ASSIGN score from the Scottish Heart Health Extended Cohort (SHHEC). Heart 2007; 93: $172-176$.

13 Foster HME, Celis-Morales CA, Nicholl BI, et al. The effect of socioeconomic deprivation on the association between an extended measurement of unhealthy lifestyle factors and health outcomes: a prospective analysis of the UK Biobank cohort. Lancet Public Health 2018; 3: e576-e585. 